\title{
Estudo de associação entre nível de atividade física, risco cardiovascular e o polimorfismo do gene da Apolipoproteína E em idosos
}

Association study among physical activity level, cardiovascular risk and the Apolipoprotein E polymorphism in elderly

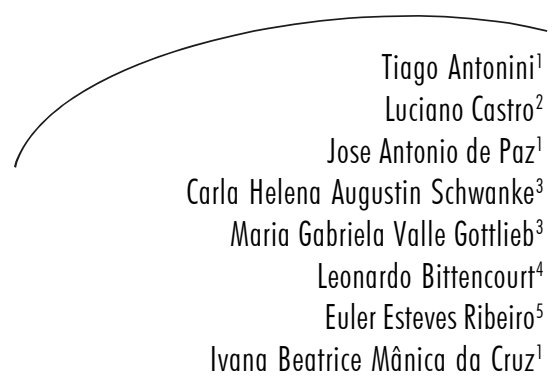

Resumo

Introdução: com o envelhecimento, ocorre perda gradual do volume da massa muscular, responsável por quase toda a perda da força nos idosos. Estudos têm mostrado que a atividade física pode diminuir a morbidade por doenças cardiovasculares, acidente vascular cerebral e aumentar a autonomia e independência. Objetivo: analisar a associação entre nível de atividade física, risco cardiovascular e o polimorfismo do gene da Apolipoproteína E em idosos. Métodos: estudo retrospectivo, observacional a partir de um banco de dados de base populacional onde foram selecionados idosos sedentários e com histórico de atividade física regular que eram portadores e não portadores do alelo e4 do gene da apolipoproteína E. Foram aplicados os seguintes questionários: (a) International Physical Activity Questionnaire (IPAC); (b) recordatório 24 horas e miniquestionário de frequência alimentar; (c) análises bioquímicas do perfil lipídico e glicêmico. Resultados: 255 idosos foram investigados; destes, 51 (20\%) eram homens e 204, mulheres (80\%). A idade média da amostra foi de 67,8 \pm 5.9 anos de idade (mínimo 60 máximo 86 anos). A frequência dos alelos foi de: alelo e3 $=0.78$, e4 $=0.16 \mathrm{e}$ e2 $=0.06 ; 45$ idosos $(17.6 \%)$ possuíam pelo menos um alelo e4 e eram sedentários, $31(12.2 \%)$ possuíam pelo menos um alelo e4 e apresentava atividade física regular (ativa), 50 (19.6\%) foram classificados como tendo os demais alelos da apoE (e2/ e3) e ativos enquanto 129 (60.6\%) eram e2/e3 e sedentários. Conclusão: a atividade física poderia ser um fator importante na atenuação dos efeitos genéticos negativos associados ao alelo e4 do polimorfismo da apoE.

\footnotetext{
Programa de Doutorado em Ciências da Atividade Física e do Esporte, Universidade de Leon, Campus de Vegazana. Leon, Espanha.

2 Faculdade de Educação Física, Pontifícia Universidade Católica do Rio Grande do Sul, Porto Alegre, RS, Brasil.

3 Instituto de Geriatria e Gerontologia, Pontifícia Universidade Católica do Rio Grande do Sul. Porto Alegre, RS, Brasil.

4 Programa de Pós-Graduação em Bioquímica Toxicológica, Universidade Federal de Santa Maria. Santa Maria, RS, Brasil.

5 Universidade Aberta da Terceira Idade, Universidade do Estado do Amazonas. Manaus, AM, Brasil.
}

E-mail.ibmcruz@hotmail.com

Palavras-Chave:

Envelhecimento. Idosos.

Atividade Física.

Polimorfismos Genéticos.

Apolipoproteínas E.

Doença Cardiovascular.

Apo E. Risco

Cardiovascular. 


\section{Abstract}

Introduction: With aging, there is gradual loss of muscle mass volume, accounting for almost all the loss of strength in the elderly. Studies have shown that physical activity can reduce morbidity from cardiovascular disease, stroke and increase the autonomy and independence. Objective: To examine the association among levels of physical activity, cardiovascular risk and gene polymorphism of $\mathrm{E}$ Apolipoprotein in the elderly. Methods: retrospective study, from an observational database on a population that selected sedentary seniors with a history of regular physical activity, carriers and noncarriers of the e4 allele of the gene of $\mathrm{E}$ Apolipoprotein. We applied the following questionnaires: (a) International Physical Activity Questionnaire (IPAC), (b) 24-hour recall and mini-food frequency questionnaire, (c) biochemical analysis of lipids and glucose levels. Results: 255 elderly were investigated. Of these, 51 (20\%) were men and 204 women $(80 \%) ; 5.9$ years of age (minimum 60 maximum $86 \pm$ ). The average age of the sample was 67.8 years). The frequency of alleles was: e 3 allele $=0.78$, e $4=0.16$ and e2 $=0.06$; 45 elderly $(17.6 \%)$ had at least one e 4 allele and were sedentary, 31 $(12.2 \%)$ had at least one e4 allele and had regular physical activity (active), 50 $(19.6 \%)$ were classified as having other alleles of the apoE (E2/E3) and active while 129 (60.6\%) were E2/E3 and sedentary. Conclusion: The physical activity could be an important factor in mitigating the negative genetic effects associated with the e4 allele of the polymorphism of apoE.
Key words: Aging. Elderlies. Physical Activity. Genetics Polymorphisms. Apo E. Cardiovascular Risk.

\section{INTRODUÇÃO}

Ao longo do envelhecimento, a perda gradual do volume da massa muscular é uma constante de tal forma que, até 50 anos, cerca de 10\% deste volume se perdem. A partir desta idade, tal perda é significativamente acelerada. Assim, a força do músculo declina em aproximadamente $15 \%$ por década dos 60 aos 70 anos; depois disso, 30\%. Embora a função intrínseca dos músculos seja reduzida com o avançar da idade, a diminuição da massa muscular é responsável por quase toda a perda da força nos idosos. O número de unidades funcionais motoras também declina com o avançar da idade, as quais são necessárias para inervar um grande número de fibras musculares. ${ }^{1}$

Por este motivo, as modificações no estilo de vida contribuem para que a quantidade de gasto energético na vida cotidiana do idoso seja aumentada, como, por exemplo, abrir janelas do carro manualmente, subir escadas, estacionar o carro em uma distância maior do que a habitual, etc. Em geral, a atividade física, sobretudo aquela que envolve estes quatro aspectos, irá: (1) melhorar a velocidade de deambulação do idoso; (2) melhorar o seu equilíbrio; (3) contribuir para a manutenção ou o aumento da densidade mineral óssea, principalmente em mulheres; (4) ajudar no controle de doenças crônicas já instaladas como diabetes, artrite, doenças coronarianas; (5) melhorar a ingestão alimentar que geralmente é problemática em pessoas idosas; (6) melhorar o sistema neurocognitivo, com ênfase na memória e na minimização de problemas depressivos comuns em idosos. $^{2}$

Alguns estudos, como os revisados por Stanner ${ }^{3}$, mostram que a participação de idosos em programas de exercício físico leva à redução de $25 \%$ de doenças cardiovasculares, $10 \%$ de acidente vascular cerebral (AVC), doença respiratória crônica e distúrbios mentais. Adicionalmente, reduz de $10 \%$ a $30 \%$ o número de indivíduos dependentes (incapazes de cuidar de si mesmos). Em termos prescritivos, exercícios físicos para pacientes idosos devem ser sucintos, mensuráveis, apropriados a sua biologia e que permita sua fácil aderência. Investigações recentes também confirmam que a atividade física é um preditor de saúde e qualidade de vida mesmo em idosos muito longevos $\left(\geq 80\right.$ anos). ${ }^{4}$ 
A relevância da atividade física ao longo do envelhecimento e na velhice parece ir além da modulação de componentes cardiorrespiratórios e fisiológicos. A mesma também permite modular ou minimizar alterações metabólicas crônicas de origem genética que, sem intervenção, determinam risco a doenças geriátricas como as cardiovasculares e as demências. ${ }^{5}$ Este é o caso do gene da apolipoproteína $\mathrm{E}$.

A apolipoproteína $\mathrm{E}$ (apoE) é um elemento protéico presente em diversas lipoproteínas, ${ }^{6}$ pertencendo aos grupos de lipoproteínas de alta densidade, muito baixa densidade e sobras de quilomícrons, também encontradas em resíduos da metabolização lipolítica. ${ }^{7}$ Responsável pelo metabolismo de lipídios, a apoE despertou interesse investigativo por estar relacionada ao transporte de colesterol em diversos tecidos. A apoE é uma proteína formada por 299 aminoácidos, sendo o seu gene polimórfico, uma vez que possui variações na sua sequência que alteram a função da proteína no organismo. O polimorfismo mais bem estudado ocorre em função de duas mutações nos aminoácidos 112 e 158 da cadeia polipeptídica que forma a apoE, onde uma cisteína é substituída por uma arginina. Estas duas variações dão origem a três alelos: e2, e3 e e4, que formam as isoformas $\mathrm{da}$ apoE conhecidas como apoE2, apoE3 e apoE4. O alelo e3 é o mais frequente nas populações, enquanto que o alelo e2 aparece em menor frequência. Uma vez que herdamos um alelo da mãe e um alelo do pai para formar nossos genes, existem seis possíveis genótipos: E2E2, E3E3, E4E4, E2E3, E3E4, E2E4. ${ }^{8}$

No caso, o alelo e4 causa uma modificação conformacional na proteína que desencadeia o aumento na concentração do LDL-colesterol plasmático. Este aumento crônico predispõe esta molécula a reagir com radicais livres e sofrer um processo de oxidação, formando uma molécula conhecida como LDL oxidada (LDL-ox). Esta molécula agora tem a capacidade de sair do sangue, atravessar o endotélio dos vasos sanguíneos e ficar no tecido conjuntivo que existe entre o endotélio e a musculatura lisa dos vasos. ${ }^{9}$
Uma quantidade extensiva de estudos tem encontrado associação positiva entre o alelo e4 do gene da apolipoproteína e com doenças cardiovasculares (para revisão, ver Kolovou \& Anagnostopoulou), ${ }^{10}$ demência do tipo Alzheimer e outras morbidades. ${ }^{11} \mathrm{O}$ grande número de associações levou ao estabelecimento de investigações para averiguar que fatores ambientais poderiam amenizar este risco genético. ${ }^{12}$ Entretanto, o número de investigações averiguando o impacto da atividade física na modulação dos fatores de risco cardiovasculares em portadores do alelo e4 ainda é muito incipiente.

Neste contexto, investigações adicionais sobre o nível de atividade física, risco cardiovascular e polimorfismo do gene da apolipoproteína $\mathrm{E}$ em idosos podem contribuir na discussão sobre a interação gene-atividade física, principalmente no perfil lipidico e outras variáveis de risco cardiovascular em idosos.

\section{METODOLOGIA}

Trata-se de estudo retrospectivo, observacional, a partir de um banco de dados de base populacional onde foram selecionados idosos sedentários e com histórico de atividade física regular que eram portadores e não portadores do alelo e4 do gene da apolipoproteína E. Nestes grupos foram comparados indicadores fisiológicos, antropométricos e nutricionais associados ao risco de doenças cardiovasculares. A população e amostra de idosos aqui investigados ( $\geq 60$ anos) fazem parte do Projeto Genesis de Pesquisa, que investiga interações genéticoambientais associadas ao envelhecimento e doenças da velhice, realizado no município de Gravataí no período de 1999 a 2005. Nesta etapa, 1.058 idosos participantes de grupos de terceira idade cadastrados na Prefeitura Municipal de GravataíRS faziam parte do projeto. Destes, 255 (24\%) indivíduos foram incluídos no presente estudo. A subamostra de idosos investigada foi selecionada a partir da presença dos seguintes critérios: (1) diagnóstico molecular prévio do polimorfismo 
da apolipoproteína E; (2) análise prévia da atividade física através da aplicação do International Physical Activity Questionnaire (IPAC) do relato de participação em atividade física regular $(>$ duas vezes por semana) nos últimos 12 meses; (3) da análise do perfil nutricional através da aplicação do recordatório 24 horas e miniquestionário de frequência alimentar; (4) análises bioquímicas do perfil lipídico e glicêmico; (5) anamnese das condições de saúde do idoso. A fim de averiguar a possível interação entre atividade física e o polimorfismo da apolipoproteína $\mathrm{E}$, foram excluídos do estudo idosos que: possuíam história prévia de doença coronariana aguda (infarto agudo do miocárdio), acidente vascular-cerebral (AVC), câncer, demência do tipo Alzheimer, hipotireoidismo e outras morbidades que poderiam interferir na análise realizada.

Quatro grupos de análises foram conduzidos no estudo. (1) inicialmente, a frequência dos genótipos, as frequências alélicas e do número de idosos com portadores de pelo menos um alelo e4 foram calculados e comparados entre idosos sedentários e com atividade física moderada. Nesta associação, verificaram-se o sexo e a idade como variáveis intervenientes; (2) a seguir foi comparado o perfil nutricional entre estes grupos de indivíduos, a fim de descartar ou avaliar o possível efeito da dieta nos resultados obtidos; (3) após estas análises, o perfil antropométrico (índice de massa corporal avaliada determinado pela divisão do peso corporal pela altura ao quadrado, IMC, $\mathrm{Kg} / \mathrm{m}^{2}$ e a circunferência abdominal), a pressão arterial sistólica e diastólica, o perfil lipídico e glicêmico foram comparados entre os grupos; (4) finalmente, a prevalência de morbidades metabólicas (hipertensão arterial sistêmica, obesidade, diabetes mellitus do tipo II e hipercolesterolemia) foi determinada e comparada. O detalhamento dos métodos utilizados para a obtenção das variáveis aqui investigadas é feito em estudos prévios do grupo de pesquisa com destaque na investigação de Schwanke et al. ${ }^{5} \mathrm{em}$ idosos longevos de origem italiana ( $>80$ anos) que também vivem no estado do Rio Grande do Sul, Brasil, e Gottlieb et al. ${ }^{2}$ nos idosos de Gravataí.
A descrição da obtenção de dados de variáveis antropométricas, bioquímicas e clínicas investigadas no estudo, previamente coletadas e disponíveis no banco de dados, é detalhada em estudos previamente publicados pela equipe de pesquisa, incluindo análises bioquímicas, nutricionais e autonomia. ${ }^{14-19}$

A atividade física foi avaliada utilizando-se o International Physical Activity Questionnaire, instrumento que foi previamente validado para o Brasil e para a aplicação em idosos por Benedetti et al. ${ }^{20}$ Adicionalmente, foram incluídos no estudo somente aqueles idosos que estavam regularmente participando em programas de atividade física para os idosos da Secretaria de Saúde do Município de Gravataí com frequência regular igual ou maior que duas vezes na semana. No grupo foram encontrados somente idosos considerados sedentários e com atividade física moderada.

As análises estatísticas foram realizadas no programa SPSS versão 12.0. Variáveis categóricas foram comparadas utilizando-se o teste estatístico do Qui-quadrado e/ou teste exato de Fisher. Variáveis quantitativas foram comparadas utilizando teste Student t. Análise do possível efeito de variáveis intervenientes foi feita através da análise de regressão logística método Backward Wald. Diferenças significativas foram consideradas quando o valor de $\mathrm{p} \leq 0.05$.

O estudo aqui apresentado representa uma análise secundária da investigação realizada por Gottlieb et al., ${ }^{13}$ que investigaram a interação entre os polimorfismos da apolipoproteína $\mathrm{E}$ o do gene da enzima superóxido dismutase dependente de manganês nos níveis de LDL-oxidada. Na ocasião, os idosos assinaram o termo de consentimento livre e esclarecido e o estudo foi conduzido dentro das normas da Resolução no 196/1996 do Conselho Nacional de Ética em Pesquisa (CONEP), que regula a pesquisa em seres humanos no Brasil.

\section{RESULTADOS}

Dos 1.058 idosos incluídos no Projeto Gravataí, 255 preencheram todos os critérios de 
inclusão e possuíam todas as informações necessárias para a realização deste trabalho. Destes, 51 (20\%) eram homens e 204 eram mulheres (80\%). A idade média da amostra foi de $67,8 \pm 5.9$ anos de idade (mínimo 60, máximo 86 anos). A idade foi similar entre os gêneros.

A frequência dos genótipos da apolipoproteína E é apresentada na tabela 1. Como esperado e descrito em outras populações, um maior número de idosos apresentava o genótipo e3e3, seguido do genótipo e3e4. A frequência do genótipo e2e2 também foi a mais baixa. Uma vez que o estudo centrou suas análises no efeito do alelo e4, os idosos foram agrupados naqueles que eram portadores de pelo menos um alelo e4 $(n=75$, $29.4 \%$ ) e naqueles que eram portadores somente de alelos e3 e/ou e4 ( $n=180,70.6 \%$ ). A frequência dos alelos foi calculada em: alelo e3 $=0.78$, $\mathrm{e} 4=0.16$ e $\mathrm{e} 2=0.06$.

Tabela 1 - Distribuição da frequência dos genótipos do polimorfismo da Apolipoproteína $\mathrm{E}$ em idosos que vivem em comunidades participantes do Projeto Gravataí. Gravataí, RS.

\begin{tabular}{lcc}
\hline Genótipos & $\mathrm{N}$ & $\%$ \\
\hline e3e4 & 156 & 61,2 \\
e2e3 & 63 & 24,7 \\
e2e3 & 24 & 9,4 \\
e4e4 & 7 & 2,7 \\
e2e4 & 5 & 2,0 \\
\hline
\end{tabular}

Em relação à atividade física, 172 (67.5\%) idosos foram considerados sedentários, enquanto que $83(32.5 \%)$ foram considerados como praticantes de atividade física moderada e regular. Não houve diferenças significativas entre o sexo nesta distribuição $(p=0.640)$. A idade média também foi similar entre os idosos sedentários e com atividade física moderada $(\mathrm{p}=0.584)$. A prevalência de riscos cardiovascular foi avaliada e os resultados são apresentados na tabela 2. Como pode ser observado, a hipertensão e a obesidade foram os riscos cardiovasculares mais prevalentes.

Tabela 2 - Prevalência de fatores de risco cardiovascular em idosos que vivem em comunidades participantes do Projeto Gravataí. Gravataí, RS.

\begin{tabular}{lcc}
\hline Variáveis & $\mathrm{N}$ & $\%$ \\
\hline Hipertensão Arterial & 165 & 64,7 \\
Tabagismo & 77 & 30,2 \\
Diabetes tipo II & 27 & 10,6 \\
Dislipidemia & 56 & 22,0 \\
Obesidade & 103 & 40,4 \\
\hline
\end{tabular}

Uma vez que o estudo utilizou dados retrospectivos, foi investigado se haveria diferença na distribuição do alelo e4 em idosos sedentários e com atividade física moderada. Os resultados apresentados na figura 1 mostraram que idosos com atividade física moderada possuíam uma frequência significativamente maior do alelo e4 em relação aos sedentários $(p=0.026)$. 


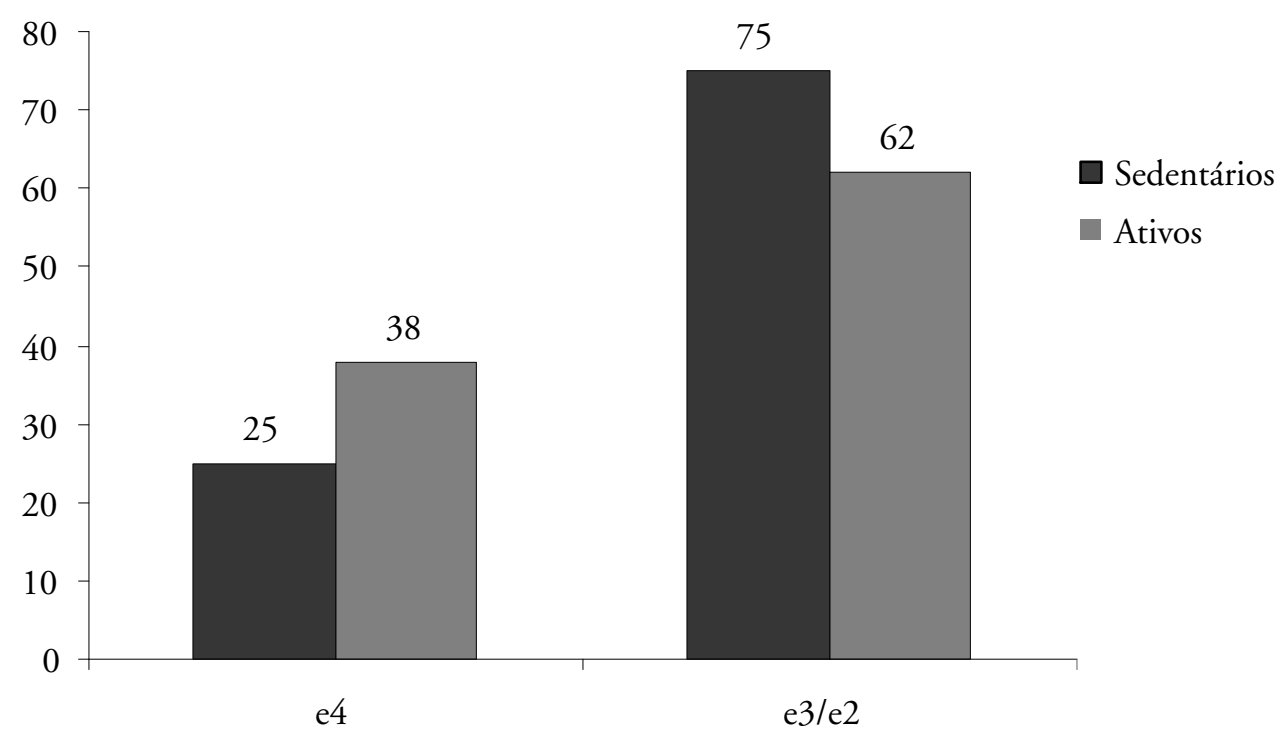

Figura 1- Comparação da distribuição do alelo e4 em idosos sedentários e com atividade física moderada.

A seguir, os idosos foram reagrupados considerando o genótipo e a atividade física. $\mathrm{Da}$ amostra investigada, $45(17.6 \%)$ possuíam pelo menos um alelo e4 e eram sedentários; 31 (12.2\%) possuíam pelo menos um alelo e4 e apresentavam atividade física regular (ativa); 50 (19.6\%) foram classificados como tendo os demais alelos da apoE (e2/e3) e ativos, enquanto 129 (60.6\%) eram e2/ e3 e sedentários.

Variáveis antropométricas, bioquímicas e fisiológicas do IMC, circunferência de cintura, perfil lipídico, glicemia, pressão arterial diastólica e sistólica foram comparados entre estes quatro grupos. Os resultados são apresentados na tabela 3 . Como pode ser observado, os níveis de HDL-colesterol em idosos portadores de pelo menos um alelo e4 e que eram considerados ativos foram significativamente maiores do que os não ativos com o mesmo padrão genético. Os níveis de HDL-colesterol dos e 4 ativos foram similares aos níveis de HDL-colesterol dos idosos e2/e3 sedentários e ativos. 
Tabela 3 - Comparação de variáveis antropométricas, bioquímicas e fisiológicas associadas ao risco cardiovascular entre idosos sedentários e ativos portadores de diferentes alelos da apolipoproteína $\mathrm{E}$. Gravataí, RS.

\begin{tabular}{lccccc}
\hline \multirow{2}{*}{ Variáveis } & \multicolumn{4}{c}{ Grupos } & P \\
\cline { 2 - 5 } & E4 sedentário & E4 ativo & $\begin{array}{c}\text { E2/E3 } \\
\text { sedentário }\end{array}$ & E2/E3 ativo & \\
\hline Colesterol (mg/dL) & $211.4 \pm 33.6^{\mathrm{a}}$ & $216.8 \pm 33.34^{\mathrm{a}}$ & $214.6 \pm 39.9^{\mathrm{a}}$ & $218.9 \pm 37.9^{\mathrm{a}}$ & 0.676 \\
Triglicerídeos (mg/dL) & $145.6 \pm 79.4^{\mathrm{a}}$ & $152.3 \pm 79.0^{\mathrm{a}}$ & $147.6 \pm 61.2^{\mathrm{a}}$ & $153.5 \pm 67.4^{\mathrm{a}}$ & 0.910 \\
HDL-col (mg/dL) & $43.0 \pm 8.8^{\mathrm{a}}$ & $47.4 \pm 8.8^{\mathrm{b}}$ & $46.0 \pm 8.6^{\mathrm{ab}}$ & $43.9 \pm 8.6^{\mathrm{a}}$ & 0.040 \\
LDL-col (mg/dL) & $137.8 \pm 37.8^{\mathrm{a}}$ & $138.9 \pm 32.4^{\mathrm{a}}$ & $140.0 \pm 34.7^{\mathrm{a}}$ & $139.6 \pm 47.5^{\mathrm{a}}$ & 0.994 \\
Glicose (mg/dL) & $104.6 \pm 30.6^{\mathrm{a}}$ & $98.3 \pm 26.7^{\mathrm{a}}$ & $100.0 \pm 22.0^{\mathrm{a}}$ & $104.5 \pm 25.9^{\mathrm{a}}$ & 0.526 \\
PAS (mmHg) & $152.1 \pm 24.1^{\mathrm{a}}$ & $155.7 \pm 27.1^{\mathrm{a}}$ & $148.5 \pm 24.8^{\mathrm{a}}$ & $152.4 \pm 28.4^{\mathrm{a}}$ & 0.680 \\
PAD (mmHg) & $80.3 \pm 11.9^{\mathrm{a}}$ & $80.0 \pm 13.5^{\mathrm{a}}$ & $77.8 \pm 12.2^{\mathrm{a}}$ & $79.0 \pm 13.2^{\mathrm{a}}$ & 0.793 \\
IMC $\left(\mathrm{Kg} / \mathrm{m}^{2}\right)$ & $28.9 \pm 4.47^{\mathrm{a}}$ & $29.7 \pm 4.9^{\mathrm{a}}$ & $28.9 \pm 4.47^{\mathrm{a}}$ & $28.9 \pm 4.2^{\mathrm{a}}$ & 0.810 \\
Cintura $(\mathrm{cm})$ & $95.7 \pm 11.8^{\mathrm{a}}$ & $96.0 \pm 11.0^{\mathrm{a}}$ & $91.9 \pm 9.6^{\mathrm{b}}$ & $91.8 \pm 10.3^{\mathrm{b}}$ & 0.049 \\
\hline
\end{tabular}

Legenda: $\mathrm{dp}=$ desvio padrão; $\mathrm{PAS}=$ pressão arterial sistólica; $\mathrm{PAD}=$ pressão arterial diastólica.; IMC- índice de massa corporal. Letras diferentes após as médias $+\mathrm{dp}$ indicam que houve diferenças significativas entre os grupos comparados em cada uma das variáveis comparadas através de análise de variância One-Way seguida de teste post hoc de Bonferroni.

A circunferência abdominal também apresentou associação com o polimorfismo da apoE. No caso, idosos portadores de pelo menos um alelo e4 apresentaram maior circunferência abdominal em relação aos portadores dos alelos e2/e4, independentemente de serem sedentários ou fisicamente ativos. Análise multivariada por regressão logística mostrou que a associação entre os níveis de HDL-colesterol mais altos em idosos portadores do alelo e4 foi independente do sexo, idade, história de diabetes do tipo II e hipertensão arterial sistêmica $($ Wald $=4,993, \mathrm{p}=0.025)$.

Uma análise adicional para averiguar se os grupos investigados possuiriam o mesmo perfil nutricional foi realizada. Os resultados são apresentados na Tabela 4. Como pode ser observado, a ingestão calórica, de proteínas, lipídios e carboidratos foi estatisticamente similar entre os grupos investigados. 
Tabela 4 - Comparação do perfil dietético entre idosos sedentários e ativos portadores de diferentes alelos da apolipoproteína E. Gravataí, RS.

\begin{tabular}{lccccc}
\hline \multicolumn{1}{c}{ Variáveis } & \multicolumn{3}{c}{ Grupos } & p \\
\cline { 2 - 4 } & E4 sedentário & E4 ativo & $\begin{array}{c}\text { E2/E3 } \\
\text { sedentário }\end{array}$ & E2/E3 ativo & \\
\hline Calorias (Kcal) & $1609 \pm 440$ & $1557 \pm 423$ & $1523 \pm 539$ & $1588 \pm 545$ & 0.842 \\
Carboidratos (\%) & $52.0 \pm 9,8$ & $50.3 \pm 7.9$ & $52.0 \pm 10.3$ & $53.9 \pm 10.2$ & 0.250 \\
Proteínas (\%) & $20.2 \pm 6.5$ & $21.2 \pm 6.5$ & $19.1 \pm 4.9$ & $19.5 \pm 5.7$ & 0.197 \\
Lipídios (\%) & $25.5 \pm 7.8$ & $26.4 \pm 6.9$ & $26.6 \pm 8.5$ & $26.2 \pm 8.8$ & 0.762 \\
\hline
\end{tabular}

Legenda: $\mathrm{dp}=$ desvio padrão; as variáveis foram comparadas através de análise de variância One-Way.

\section{DISCUSSÃO}

Neste estudo retrospectivo, foi investigado se a frequência do alelo e4 do gene da apolipoproteína $\mathrm{E}$, que é considerado risco para diversas doenças, incluindo as cardiovasculares, seria similar entre idosos sedentários e com atividade física moderada. Adicionalmente, foi analisado o possível efeito da interação entre o polimorfismo da apoE e atividade física em marcadores plasmáticos (lipídicos e glicêmicos), antropométricos e da pressão arterial sistólica. Os resultados obtidos mostraram uma frequência maior do alelo e4 no grupo dos idosos ativos do que no dos idosos sedentários, e a interação entre o gene da apoE e atividade física nos níveis de HDL-colesterol. No caso, idosos portadores do alelo e4 considerados como tendo uma atividade física diária moderada apresentaram níveis altos de $\mathrm{HDL}$ colesterol do que os sedentários.

Apesar da relevância deste tipo de estudo, investigações prévias sobre o tema são relativamente poucas. Um estudo que pode servir de referência foi o conduzido por Corella et al. ${ }^{21}$ em espanhóis. Apesar de os autores usarem uma amostra grande (966), a variação da idade da amostra também foi grande (18 a 66 anos). Isto sugere que a amostra aqui utilizada, de 255 indivíduos, é satisfatória para a análise, uma vez foi composta apenas de indivíduos com idade igual ou acima de 60 anos. Os autores observaram que a frequência do alelo e4 foi de 0.06 , enquanto na nossa amostra o alelo e4 presente na população apresentou uma frequência de 0.16 . Esta variação nas frequências do alelo e4 entre diversas populações já foi anteriormente determinada. ${ }^{22}$ Schwanke et al. ${ }^{5}$ investigando uma população de idosos longevos que vivem no sul do Brasil e que eram de origem italiana, encontraram uma frequência do alelo e4 de 0.11. Esta frequência foi similar ao descrito na Itália. ${ }^{23}$

Ao contrário de outros trabalhos, como o de Schwanke et al., ${ }^{5}$ não se observou na amostra aqui investigada associação entre níveis lipídicos, glicêmicos, antropométricos e de pressão arterial sistólica entre idosos com diferentes genótipos da apolipoproteína E. A prevalência de morbidades cardiometabólicas também foi similar, considerando-se tanto o polimorfismo da apoE quanto a atividade física. Estes resultados podem estar associados ao fato de que foram excluídos da amostra indivíduos com morbidades prévias, como infarto agudo do miocárdio, câncer, demências, etc., que poderiam apresentar alterações nestas variáveis. Por outro lado, quando a amostra foi agrupada considerando a presença de pelo menos um alelo e4 e o tipo de categoria do idoso em relação à atividade física, observouse aumento nos níveis de HDL-colesterol no grupo dos idosos portadores do alelo e4 e considerados fisicamente ativos. 
Em seu estudo, Corella et al. ${ }^{21}$ encontraram interação relacionada ao sexo dos sujeitos investigados. No caso, mulheres que consumiam maior quantidade de álcool, mas eram portadoras dos alelos e3 e e2, apresentaram menores concentrações plasmáticas de LDL-colesterol do que as mulheres e4. Já os autores observaram que homens com maior nível de atividade física e portadores do alelo e4 apresentavam níveis elevados de HDL-colesterol. Já o Cardiac Study encontrou resultado contrário: mulheres portadoras do alelo e4 fisicamente ativas apresentavam níveis de HDLcolesterol mais altos. ${ }^{24}$

Como foi comentado por esses autores na discussão de seus resultados, diversos fatores, como o fumo, consumo alcoólico, atividade física e educação poderiam interagir com o polimorfismo da apoE, afetando assim os níveis lipídicos. Entretanto, poucos estudos têm focado este tipo de análise, como é o caso da investigação aqui apresentada. Por este motivo, a comparação dos resultados obtidos com análises prévias em outras populações pode ser considerada relevante.

Considerando os primeiros estudos sobre o tema, realizados cerca de 15 anos atrás, praticamente nenhum apontou para uma associação direta entre apoE e níveis de HDLcolesterol. Entretanto, uma meta-análise realizada ainda em 1992 por Dallongeville et al. ${ }^{25}$ Mostrou que já e havia encontrado que as concentrações do HDL-colesterol poderiam ser influenciadas pelo genótipo da apoE. Um estudo anterior conduzido no Brasil por outro grupo de pesquisa em 672 indivíduos, com idade entre 18 a 80 anos, também observou menores níveis de HDLcolesterol em portadores do genótipo e3e4 em relação aos genótipos e3e3 e e2e3. ${ }^{26}$ Outra investigação na Índia realizada por Singh et al. ${ }^{27}$ também observou valores baixos de HDLcolesterol em indivíduos com o genótipo e3e4.

Uma recente investigação realizada em 985 indivíduos sul-asiáticos, chineses e canadenses de origem europeia investigou o polimorfismo da apoE, concentrações plasmáticas e presença de aterosclerose na artéria carótida. Os autores observaram que as frequências dos alelos e2, e3 e e4 variavam entre os grupos étnicos investigados e que houve associação entre o polimorfismo da apoE e níveis plasmáticos, com exceção dos triglicerídeos. O alelo e4 foi associado com níveis superiores de LDL-colesterol e níveis mais baixos de HDL-colesterol. Fatores da dieta e do estilo de vida influenciaram várias características investigadas, incluindo os níveis de HDLcolesterol. A principal conclusão dos autores foi que o polimorfismo da apoE realmente está associado com os níveis plasmáticos lipídicos e de lipoproteínas com pouca influência interétnica nesta associação..$^{28}$ Os autores comentaram que, predominantemente, o consumo de álcool e a atividade física apresentavam uma associação moderada nos níveis de HDL-colesterol.

Como dentro das variáveis do estilo de vida a dieta também tem influência muito grande no perfil lipídico, uma análise adicional comparando o perfil de ingestão calórica e de macronutrientes estimados a partir do recordatório 24 horas foi conduzida. Os resultados mostraram um perfil similar de ingestão entre os quatro grupos investigados, sugerindo ser a associação entre níveis altos de HDL-colesterol e atividade física em idosos portadores do alelo e4 pouco influenciada pela nutrição.

Outra questão importante para ser discutida está relacionada com o maior número de idosos portadores de pelo menos um alelo e4 no grupo de atividade física moderada. O gene da apolipoproteína está associado à longevidade, já que indivíduos portadores do alelo e 2 têm mais chance de chegar a idades avançadas nonagenárias e centenárias. Assim, no grupo dos fisicamente ativos, o maior percentual de portadores do alelo e4 em relação aos sedentários poderia ser atribuída ao efeito positivo da atividade física na saúde destes indivíduos, que levou os mesmos a apresentarem uma carga de morbi-mortalidade menor. Investigações complementares que comprovem esta hipótese precisarão ser conduzidas.

Com base nestas evidências prévias e nos resultados aqui obtidos, pode-se sugerir que o efeito da atividade física nos indivíduos 
portadores do alelo e4 seria potencialmente benéfico, uma vez que o aumento nos níveis de HDL-colesterol são relevantes na saúde cardiovascular.

É claro que o presente estudo possui limitações metodológicas entre as quais podemos citar: (1) por ser um estudo retrospectivo, não foi possível incluir outras informações e variáveis que poderiam auxiliar na análise dos resultados; (2) a inclusão de participantes que estavam recebendo medicamentos para diabetes, hipertensão ou hipercolesterolemia pode alterar os níveis fisiológicos lipídicos e confundir a análise de associações entre o perfil lipídico, atividade física e o polimorfismo da apoE. Entretanto, esta é uma limitação presente em praticamente todos os trabalhos realizados, como por exemplo, o de Burman et al., ${ }^{28}$ recentemente publicado na revista Atherosclerosis; (3) o tamanho da amostra poderia ser considerado relativamente pequeno. Porém,

\section{REFERÊNCIAS}

1. Mcdermott AY, Mernitz H. Physical activity and risk of endometrial cancer: a populationbased prospective cohort study. [ acesso em 6 Nov 2006] Disponível em:http://www.aafp.org/ afp/20060801/437.html

2. Woo J. Relationships among diet, physical activity and other lifestyle factors and debilitating diseases in the elderly. Eur J Clin Nutr 2000 Jun; 54 Suppl 3:S143-7.

3. Stanner S. Diet and lifestyle measures to protect the ageing heart. Br J Community Nurs 2009 May;14(5):210

4. Stessman J, et al. Physical activity, function, and longevity among the very old. Arch Intern Med. 2009 Sep 14;169(16):1476-83.

5. Schwanke CHA, et al. Análise da Associação entre Polimorfismo do Gene da Apolipoproteína E e Fatores de Risco Cardiovasculares em Idosos Longevos. Arq Bras de Cardiologia 2002; 78 (6) :561-70.

6. Novak EM, Bydlowsi SP. Biologia Molecular das Dislipidemias :variação Genética das Apolipoproteínas. Arq Bras de Cardiologia 1996; 67 (6).

7. Brandão AC, et al. Polimorfismo genético da apolipoproteína $\mathrm{E}$ na doença arterial periférica. J.1 Vas Bras 2004; 3(4): 317-22. como idosos com alta carga de morbidade foram excluídos do estudo, esta condição diminuiu a variância associada à investigação.

\section{CONCLUSÃO}

Independentemente das limitações do estudo e da necessidade de investigações complementares, sabe-se que um grande número de estudos tem atribuído modificações benéficas nos níveis e composição química das frações e subfrações do HDL-colesterol e LDL-colesterol através do exercício físico, principalmente do treinamento aeróbio. Deste modo, os resultados aqui obtidos indicam fortemente que, além dos benefícios gerais de saúde física e mental relacionados à atividade física nos idosos, a atividade física poderia ser um fator importante na atenuação dos efeitos genéticos negativos associados ao alelo e4 do polimorfismo da apoE.
8. Angelopoulos TJ, Lowndes J. ApoE genotype: impact on health, fitness and nutrition. Rev Nutr Diet 2008;98:77-93.

9. Boekholdt $\mathrm{M}$, et al. Common variants of multiple genes that control reverse cholesterol transport together explain only a minor part of the variation of HDL cholesterol levels. Clin Genet 2006; 69 : 263-270.

10. Kolouvou GD, Anagnostopoulou KK. Apolipoprotein E polymorphism, age and coronary heart disease. Rev Ageing Res 2007 Aug;6(2):94-108.

11. Jofre-Monseny 1, Minihane AM, Rimbach G. Impact of apoE genotype on oxidative stress, inflammation and disease risk. Mol Nutr Food Res 2008 Jan;52(1):131-45.

12. Elbaz A, Dufouil C, Alperovitch A. Interaction between genes and environment in neurodegenerative diseases. C R Biol 2007 Apr; $330(4): 318-28$.

13. Gottlieb, MGV, et al. Association among oxideized LDL levels, MnSOD, apolipoprotein E polymorphisms and cardiovascular risk factors in a South Brazil region population. GRM 2005; 4 (4): 691703. 
14. Prado-Lima PS, et al. Human food preferences are associated with a 5-HT2 Aserotonergic receptor polymorphism. Molecular Psychiatry 2006;11: 889-891.

15. Montano MAE, et al. Association between manganese superoxide dismutase (MnSOD) gene polymorphism and elderly obesity. Molecular and Cellular Biochemistry 2009: 0071-z

16. Jobim PPF, et al. The serotonin-2A receptor T102C polymorphism is associated with age in a Brazilian population. Braz J Med 2008; 41:1018-1023.

17. Manica-Cattani MF, et al. Association between interleukin-1 beta polymorphism (+3953) and obesity. Mol Cell Endocrino 20091; 309: 1-2.

18. Schwanke, CHA, et al. Is there an association between T102C polymorphism of the serotonin receptor $2 \mathrm{~A}$ gene and urinary incontinence? Braz J. Med 2007; 40:1-2

19. Noronha J P, et al. Association between T102C polymorphism of serotonin 2 a receptor gene and urinary incontinence in older women. JIM; 57; 1-09.

20. Benedetti TRB. Reprodutibilidade e validade do questionário Internacional de Atividade Física (IPAQ) em homens idosos. Rev Bras Med do Esporte 2007 ;Jan/Fev;13 (1). Corella D, et al

21. Corella D, et al. Environmental factors modulate the effect of the APOE genetic polymorphism on plasma lipid concentrations: ecogenetic studies in a Mediterranean Spanish population. Metabolism 2001 Aug;50(8):936-44.

22. Gerdes LU, et al. Apolipoprotein and polymorphism in a Danish population compared to findings in 45 other study populations around the World. Genet Epidemiology 1992; 9: 155-167.

23. Bader G, et al, Apolipoprotein E polymorphism is not associated with longevity or disability in a sample of italian octo-and nonagenarians, Gerontology 1998; 44: 293-9.

24. Howard BV, et al. Association of apolipoprotein E phenotype with plasma lipoproteins in African-Amarican and White Young adults. Am. J. Epidemiology 1998;1480-859.

25. Dallongeville S, Lussier-Cacan, Davignon J . Modulation of plasma triglyceride levels by apoE phenotype: a meta-analysis. J Lipid Res $1992 ; 33$ : 447-454.

26. Martins MC, et al. Influência dos Polimorfismos da APOE em alguns Fatores de risco de Aterosclerose. Acta Médica Portuguesa 2008; 21: 433-440.

27. Singh PP, et al. Apolipoprotein E polymorphism and its relation to plasma lipids in coronary heart disease. Indian J Med Sci 2008.]

28. Burman D, et al. Relationship of the ApoE polymorphism to plasma lipid traits among South Asians 
